\title{
Propagación y establecimiento de lavanda (Lavandula angustifolia Mill.) bajo malla sombra
}

\author{
Propagation and establishment of lavender \\ (Lavandula angustifolia Mill.) under shade cloth \\ Diana Miriam Mc Caughey-Espinoza ${ }^{1}$, Gloria Irma Ayala-Astorga ${ }^{1}$, \\ Génesis V. Buitimea-Cantúa², Nydia E. Buitimea-Cantúa², Andrés Ochoa-Meza ${ }^{3}$
}

\section{RESUMEN}

La planta de lavanda es una especie de arbusto que tiene una demanda importante en la industria cosmética, aromaterapia, entre otros ámbitos. El objetivo de esta investigación fue estudiar la propagación de esquejes de lavanda (Lavandula angustifolia Mill) y su crecimiento bajo malla sombra en el estado de Sonora. Se utilizaron dos tratamientos de cultivo (suelo y pet moss como sustrato) con 4 repeticiones de 25 esquejes. Se evaluó el porcentaje de enraizamiento de los esquejes, porcentaje de sobrevivencia al trasplante de los esquejes, altura y diámetro de cobertura de las plantas. El porcentaje de enraizamiento de los esquejes cultivados en suelo fue 4.14 veces mayor comparado con los esquejes cultivados en pet moss. Por otra parte, el porcentaje de sobrevivencia al trasplante de los esquejes ya enraizados fue del $96 \%$ en ambos tratamientos. Los esquejes cultivados en suelo presentaron 1.06 veces mayor altura y $1.08 \mathrm{~cm}$ veces mayor diámetro de cobertura que los esquejes cultivados en pet moss. Las plantas de lavanda de ambos tratamientos mostraron floración al mes de ser trasplantados. Además, la longitud de las raíces se afectó por el tamaño de esqueje y la longitud de la raíz principal fue influenciada por la cantidad de raíces secundarias. No se presentaron plagas ni enfermedades en las plantas de lavanda en el área de estudio. La lavanda crecida bajo malla sombra puede ser un cultivo con un gran potencial productivo para el noroeste de México.

Palabras clave: lavanda, propagación, esquejes, suelo, pet moss.

\begin{abstract}
The lavender plant is a kind of shrub that presents an important demand in the market of cosmetic, aromatherapy, among others. The objective of this research was to study the propagation of lavender cuttings (Lavandula angustifolia Mill) and their growth under shade cloth. Two cultivation treatments were used (soil and pet moss as a substrate) with 4 repetitions of 25 cuttings. The percentage of rooting of the cuttings, percentage of survival to the transplantation of the cuttings, height and diameter of plant cover were evaluated. The rooting percentage of cuttings grown in soil was 4.14 times higher than cuttings grown in pet moss. On the other hand, the survival percentage after transplantation of the rooted cuttings was $96 \%$ in both treatments. The cuttings cultivated in soil presented 1.06 times greater height and $1.08 \mathrm{~cm}$ greater diameter of coverage than the cuttings cultivated in pet moss. The lavender plants of both treatments showed flowering a month after being transplanted. Furthermore, the root length was affected by the cutting size and the length of the main root was influenced by the amount of secondary roots. There were no pests or diseases in the lavender plants in the study area. Therefore, lavender grown under shade cloth can be a crop with great productive potential for northwestern Mexico.
\end{abstract}

Keywords: lavender, propagation, cuttings, soil, pet moss.

\section{Introducción}

El estado de Sonora cuenta con especies aromáticas importantes en sus diferentes ecosistemas, entre las cuales se encuentran el orégano, la salvia, damiana y valeriana. Es necesario señalar que la agricultura está buscando otros enfoques agromercantiles aunado a la rotación y establecimiento de nuevos cultivos

\footnotetext{
1 Departamento de Investigaciones Científicas y Tecnológicas de la Universidad de Sonora. México. Sonora, Hermosillo..

2 Tecnológico de Monterrey, Centro de Biotecnología-FEMSA, Escuela de Ingeniería y Ciencias, Campus Monterrey.

3 Departamento de Agricultura y Ganadería. Universidad de Sonora. México. Sonora, Hermosillo.

* Autor por correspondencia: nebc@tec.mx
}

Fecha de Recepción: 18 de Octubre, 2019.

Fecha de Aceptación: 5 de Noviembre, 2020. 
que requieran menos agua para su producción. En los últimos años el interés por las hierbas aromáticas medicinales se ha venido incrementando paulatinamente entre los recolectores, productores, industrias transformadoras, instituciones públicas y/o privadas y consumidores (Azizi et al., 2006).

México cuenta con zonas con alto potencial para la producción de hierbas finas, aromáticas y medicinales, y su creciente demanda las ha convertido en productos con un nicho de mercado rentable y en expansión (Juárez-Rosete et al., 2013). Los principales productores de hierbas aromáticas de exportación que cumplen con los protocolos de Buenas Prácticas Agrícolas o Certificación orgánica están en los estados Morelos, Baja California Sur, Baja California, Estado de México, Nayarit, Oaxaca, Puebla y Tlaxcala (Pérez, 2009).

En la búsqueda de nuevas alternativas de cultivo dentro del ámbito agroindustrial, alimenticio, medicinal, entre otros, la lavanda podría cubrir expectativas importantes para algunas regiones de nuestro estado. La lavanda (Lavandula angustifolia Mill) es un arbusto y sus flores son atractivas para algunos insectos como las abejas. Su aceite esencial presenta compuestos activos importantes como linalol, geraniol, borneol, los cuales tienen principal efecto en el sistema nervioso central ayudando a la relajación de personas que viven en constante estrés en la sociedad actual (Celedón y Lerdon, 2014). Además, la lavanda es una planta de bajos requerimientos de agua, lo que la convierte en una excelente alternativa de cultivo en el estado de Sonora.

La macropropagación vegetativa o asexual es la multiplicación de una planta a partir de una célula, un tejido o un órgano. Se producen individuos a partir de secciones vegetativas, obteniendo clones de la planta y conservando las características propias de cada individuo (Hartmann et al., 2011). Dentro de la multiplicación asexual, la propagación por estacas es la técnica más común para las especies de importancia forestal, florística, agrícola y agroindustrial (Dole y Hamrick, 2006). Esta técnica permite la propagación en un espacio pequeño, con la ventaja de adquirir suficiente material a partir de una sola planta (Ocampo y Valverde, 2000). Por lo tanto, el objetivo de este estudio fue estudiar la propagación de esquejes de lavanda (Lavandula angustifolia Mill) y su crecimiento bajo malla sombra en el estado de Sonora.

\section{Materiales y métodos}

Ubicación del área de estudio. El trabajo de investigación se llevó a cabo en el ciclo de primaveraverano del 2017, en el vivero del Departamento de Investigaciones Científicas y Tecnológicas de la Universidad de Sonora, ubicado en las coordenadas $29^{\circ} 05^{\prime} 00^{\prime \prime} \mathrm{N} 110^{\circ} 57^{\prime} 51^{\prime \prime} \mathrm{O}$.

Área seleccionada. Se seleccionó un área de $40 \mathrm{~m}^{2}$, la cual se encontraba bajo una malla sombra de $80 \%$ de luz con un tipo de suelo arcilloso y escasa materia orgánica.

Material vegetal. El material vegetal se colectó con unas tijeras para podar desinfectadas previamente con cloro al $10 \%$. Los esquejes se recogieron de plantas adultas que tenían cuatro meses sembradas directamente en el suelo del vivero bajo malla sombra.

Labores agronómicas. Se realizó deshierbe manual, se removió el suelo y se levantaron los surcos.

Instalación del sistema de riego. Considerando que es una planta que requiere de humedad y en el estado de Sonora se presentan bajas precipitaciones al año, se instaló un sistema de riego por goteo. De este modo se generó un bulbo de humedad en la parte radicular de las plantas. El gasto que se originó por planta fue de $1 \mathrm{lph}$ por gotero de acuerdo a las temperaturas presentes, proporcionándoles media hora de riego cada dos días.

Densidad de plantas. Para esta investigación se manejó una densidad de plantas de 84 plantas. La distancia entre cada planta fue de $50 \mathrm{~cm}$ y 1 metro de distancia entre cada surco.

Tratamiento. Para observar el comportamiento del tiempo de enraizamiento de los esquejes se utilizaron dos tratamientos como sustratos: suelo y pet moss.

Trasplante de los esquejes al suelo y macetas. Los esquejes que se utilizaron para este estudio medían aproximadamente 10 a $12 \mathrm{~cm}$ de longitud. Se colocaron previamente en el suelo y en charolas con pet moss.

\section{Parámetros a evaluar}

El porcentaje de enraizamiento se evaluó en las plantas de lavanda cultivadas en el suelo y pet moss de acuerdo a Garay y Echeverría (2013). El porcentaje de sobrevivencia al trasplante se observó en las plantas de lavanda cultivadas en el suelo y pet moss (Mc Caughey-Espinoza et al., 2019). 
Mediciones dasométricas. Las mediciones de altura y diámetro de copa se realizaron en 10 ejemplares de plantas de lavanda cultivadas en el suelo y pet moss. Se utilizó una cinta métrica graduada en cm (Meza y Osuna, 2003). Las mediciones se llevaron a cabo a los 4 meses después del trasplante.

Longitud de raíz principal. Las raíces fueron previamente lavadas para eliminar el sustrato (suelo o pet moss). Se midió la longitud de la planta de lavanda desde la base del tallo hasta el ápice de la raíz más larga, utilizando una cinta métrica graduada en cm (Garay y Echeverría, 2013). Se consideraron únicamente las raíces primarias sin tener en cuenta las secundarias y terciarias (Garay y Echeverría, 2013).

Plagas y enfermedades presentes en las plantas. Se evaluó durante 6 meses, el tiempo en que se realizó este estudio.

\section{Diseño experimental y análisis estadístico}

Se utilizó un diseño completamente al azar y la variable a evaluar fue el porcentaje de enraizamiento de los esquejes (en suelo y pet moss). Cada tratamiento presentó cuatro repeticiones con 25 esquejes (100 esquejes por tratamiento). Se realizó un análisis de varianza (ANDEVA) con un nivel de significancia de $p<0,05$. Se hizo un análisis de comparación de medias por Tukey-Kramer con un alfa de 0,05\% y análisis de correlaciones de Pearson. Estos análisis se llevaron a cabo con el paquete estadístico JMP 5.0.1a (JMP, 2011).

\section{Resultados y discusión}

\section{Porcentaje de enraizamiento de esquejes}

El porcentaje de enraizamiento de los esquejes se afectó significativamente $(p<0,05)$ por el tipo de sustratos (suelo y pet moss). Las plantas de lavanda con sustrato de suelo presentaron un mayor porcentaje de enraizamiento (87\%) que las cultivadas en pet moss (21\%). El uso de pet moss para raizar esquejes de lavanda (Lavandula angustifolia Mill) no fue adecuado al mostrar cierta porosidad en el sustrato, lo que puede atribuirse a la presencia de aire en la parte basal. En la Figura 1 se observa el porcentaje de enraizamiento de las plantas de lavanda cultivadas en el suelo y pet moss. Este comportamiento puede deberse a la longitud de los esquejes, los cuales indicaron que la cantidad de meristemos principales son el sitio de la síntesis de auxinas, encargadas de estimular los procesos de rizogénesis (Salisbury y Ross, 1994; Marschner, 2002; Herrera et al., 2007). A mayor enraizamiento se produce el movimiento denominado transporte polar de auxinas, ocasionando que estas se desplacen más rápido hacia la parte inferior de los tallos (Bandurski et al., 1993; Lemes-Hernández et al., 2001).

El enraizamiento de las plantas se favorece debido a varios factores como reducir la transpiración de los esquejes, optimizar el proceso de fotosíntesis, tipo de sustratos y reguladores de crecimiento que faciliten la formación de raíces (Ruiz y Mesén, 2010; Hartmann et al., 2011). En este estudio no se eliminaron las hojas de los esquejes, lo que favoreció el enraizamiento, proporcionando auxinas,

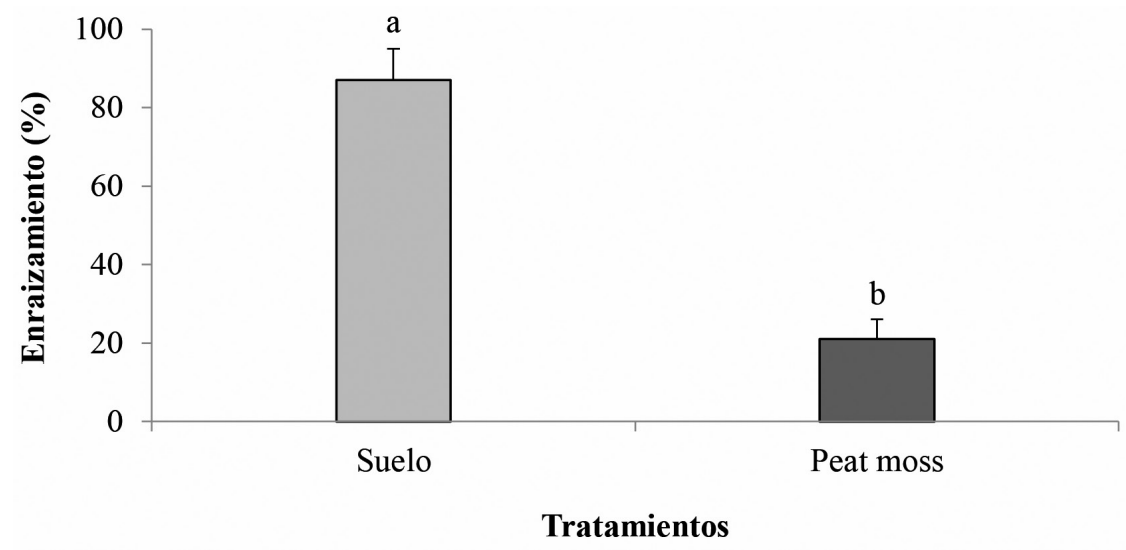

Figura 1. Porcentaje de enraizamiento de los esquejes de lavanda en diferentes sustratos (suelo y pet moss). 
vitaminas, carbohidratos, nitrógeno orgánico y cofactores que son requeridos para echar raíces. Por otro lado, también contribuyen a la desecación del esqueje al ofrecer una mayor superficie para que ocurra la transpiración (Hartmann et al., 2011).

La edad del tejido vegetal es muy importante al utilizar el sistema de esquejes. Garay y Echeverría (2013) recomiendan usar esquejes que provengan de material vegetal joven. Igualmente, Kochhar et al. (2008) señalaron que la edad del tejido vegetal, época de recolección, composición bioquímica, posición de la estaca en la planta madre y la presencia de hojas y yemas influyen en la respuesta al enraizamiento. Algunos estudios han mostrado la capacidad de la formación de raíces en diferentes posiciones de la misma rama y la importancia de los carbohidratos y hormonas vegetales (Swamy et al., 2002; Kochhar et al., 2008).

Se han realizado varias investigaciones utilizando el método de esquejes para cultivos de Sacha Inchi (Plukenetia volubilis L.), azul de mata (Justicia tinctoria (OERST.) D.N. Gibson, fam. acanthaceae), lulo silvestre (Solanum hirtum Vahl, S. marginatum L.f., S. sessiliflorum Dun, S. mammosum L. y S. umbellatum Mill.) y romero (Rosmarinus officinalis L.) con diferentes reguladores de crecimiento vegetal auxínico sintético como ácido indolbutírico (AIB) y naftalenacético (ANA) (Ruiz y Mesén, 2010; Solís et al., 2015; Ordóñez et al., 2012; Herrera et al., 2007). Ruiz y Mesén (2010) evaluaron el efecto de diferentes concentraciones de ácido indolbutírico y tipo de esqueje en el enraizamiento de Sacha Inchi (Plukenetia volubilis L.), obteniendo un alto porcentaje de enraizamiento (80\%). Recientemente, Solís et al. (2015) estudiaron el enraizamiento de azul de mata (Justicia tinctoria (OERST.) D.N. Gibson, fam. Acanthaceae) bajo un ambiente protegido, utilizando el ácido indolbutírico en tres concentraciones (500, 1000 y $1500 \mathrm{ppm})$, estacas de diferente longitud $(6,10$ y $14 \mathrm{~cm}$ ) y distintos sustratos (arena de río, fibra de coco o carbón de granza de arroz). Se obtuvo un mayor enraizamiento (92\%) con estacas de $6 \mathrm{~cm}$ de longitud usando como sustrato arena y concentraciones de ácido indolbutírico de 1000 y 1500 ppm. Ordóñez et al. (2012) analizaron la propagación vegetativa mediante esquejes de lulo silvestre (Solanum hirtum Vahl, S. marginatum L.f., S. sessiliflorum Dun, S. mammosum L. y S. umbellatum Mill), utilizando ácido naftalenacético, y se obtuvieron bajos porcentajes de enraizamiento en $S$. sessiliflorum $(62,5 \%)$ y $S$. marginatum $(61,9 \%)$ comparados con los reportados en Sacha Inchi y azul de mata cuando se usó el ácido indolbutírico. Sin embargo, en este estudio, en ambos tratamientos (suelo y pet moss) no se utilizaron reguladores de crecimiento vegetal auxínico sintéticos y fertilizantes químicos, logrando un mayor enraizamiento en los esquejes de lavanda (sustrato de suelo). Este comportamiento fue similar al observado en Sacha Inchi y azul de mata. Los esquejes de lavanda enraizados en el suelo directamente presentaron raíces más firmes y vigorosas que los esquejes enraizados en pet moss, los cuales produjeron raíces artificiales y débiles (Figura 2 A-B). Estas características de la vigorosidad de las raíces son un factor importante para asegurar la sobrevivencia al trasplante.

\section{Sobrevivencia al trasplante}

Las plantas (esquejes de lavanda) enraizadas en maceta con pet moss y las cultivadas en el suelo presentaron un $96 \%$ de sobrevivencia al trasplante. Este comportamiento fue muy aceptable para estas especies de plantas, porque el trasplante se llevó a cabo en el mes de marzo, cuando las temperaturas oscilaron entre 31 y $33{ }^{\circ} \mathrm{C}$. En la Figura 3 A-B se observan las plantas de lavanda establecidas para su climatización y se comprueba que esta especie puede adaptarse satisfactoriamente a las condiciones de temperatura presentes en la región.

El alto porcentaje de sobrevivencia al trasplante de las plantas de lavanda se puede atribuir a que se produjo un microclima y se mantuvo la humedad por más tiempo en la parte radicular de los esquejes, mediante la colocación de un vaso térmico abierto por la parte superior (Figura $3 \mathrm{~A}$ ). Así se conservóo una humedad constante en los primeros tres días posteriores al trasplante para asegurar la sobrevivencia de las nuevas plantas de lavanda (Mc Caughey-Espinoza et al., 2017).

\section{Mediciones dasométricas}

A los cuatro meses del trasplante y adaptación de los esquejes de lavanda, se les midió la altura. Las nuevas plantas de lavanda producidas por esquejes y enraizadas en el suelo presentaron alturas medidas de la base del tallo a la parte apical de las plantas de $27,05 \mathrm{~cm}$, mientras que en 
A)

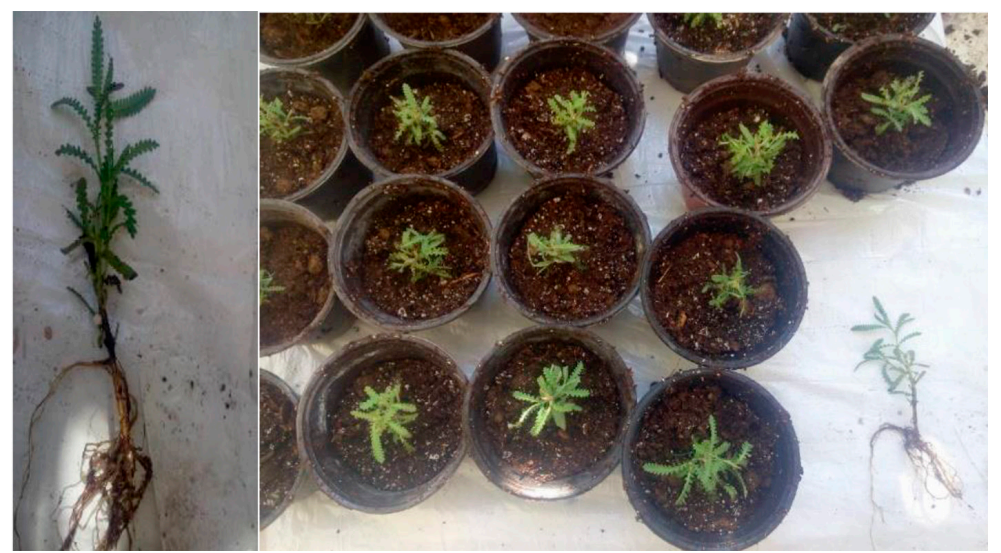

B)

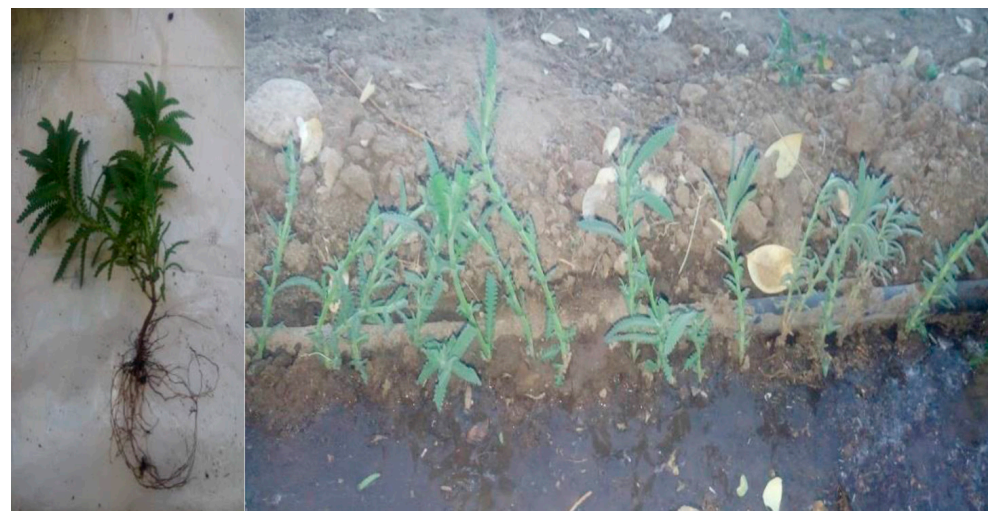

Figura 2. Esquejes de lavandas enraizadas: A) Pet moss y B) Suelo.
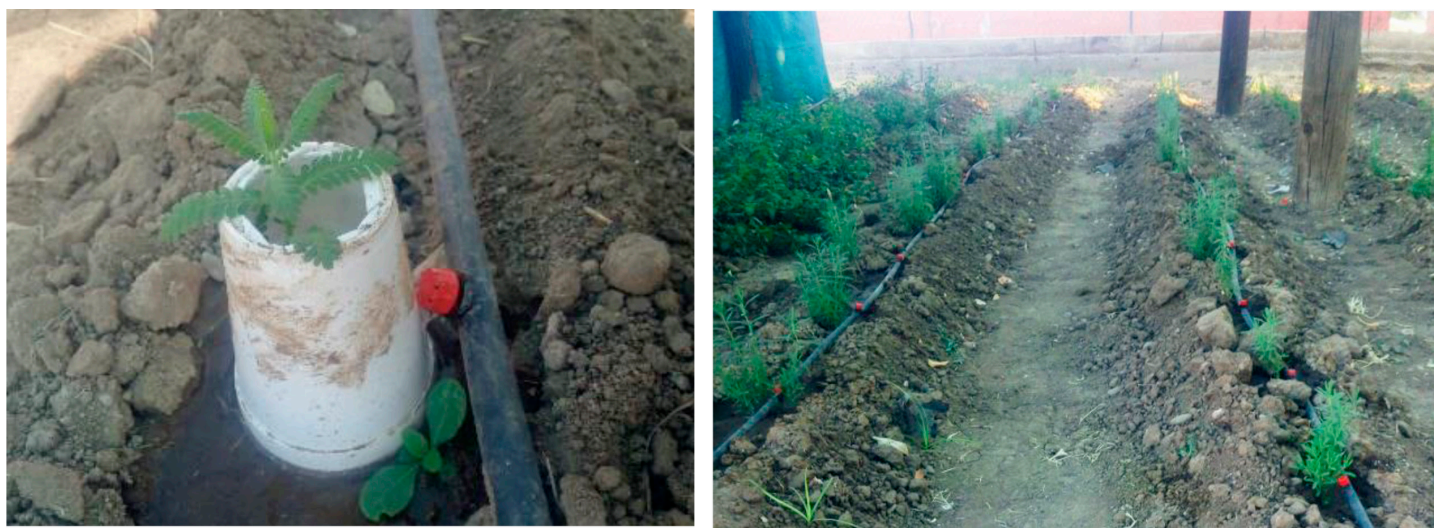

Figura 3. A) Colocación de vaso térmico a los esquejes trasplantados. B) Plantas de lavanda cultivada en el suelo.

los esquejes producidos en pet moss su altura fue de 25,44 cm (Figura 4A; Figura 5A). Al mes del trasplante de los esquejes de lavanda que fueron previamente enraizados en pet moss y en suelo mostraron floración (Figura 5C). Este proceso fisiológico que se produjo en las nuevas plantas de lavanda es importante porque se pueden colectar semillas y nos garantiza que son plantas vigorosas.

Las nuevas plantas de lavanda enraizadas en suelo presentaron diámetros de copa de 16,02 cm, mientras que las que fueron enraizadas en pet moss alcanzaron diámetros de 14,81 cm (Figura 4B; 

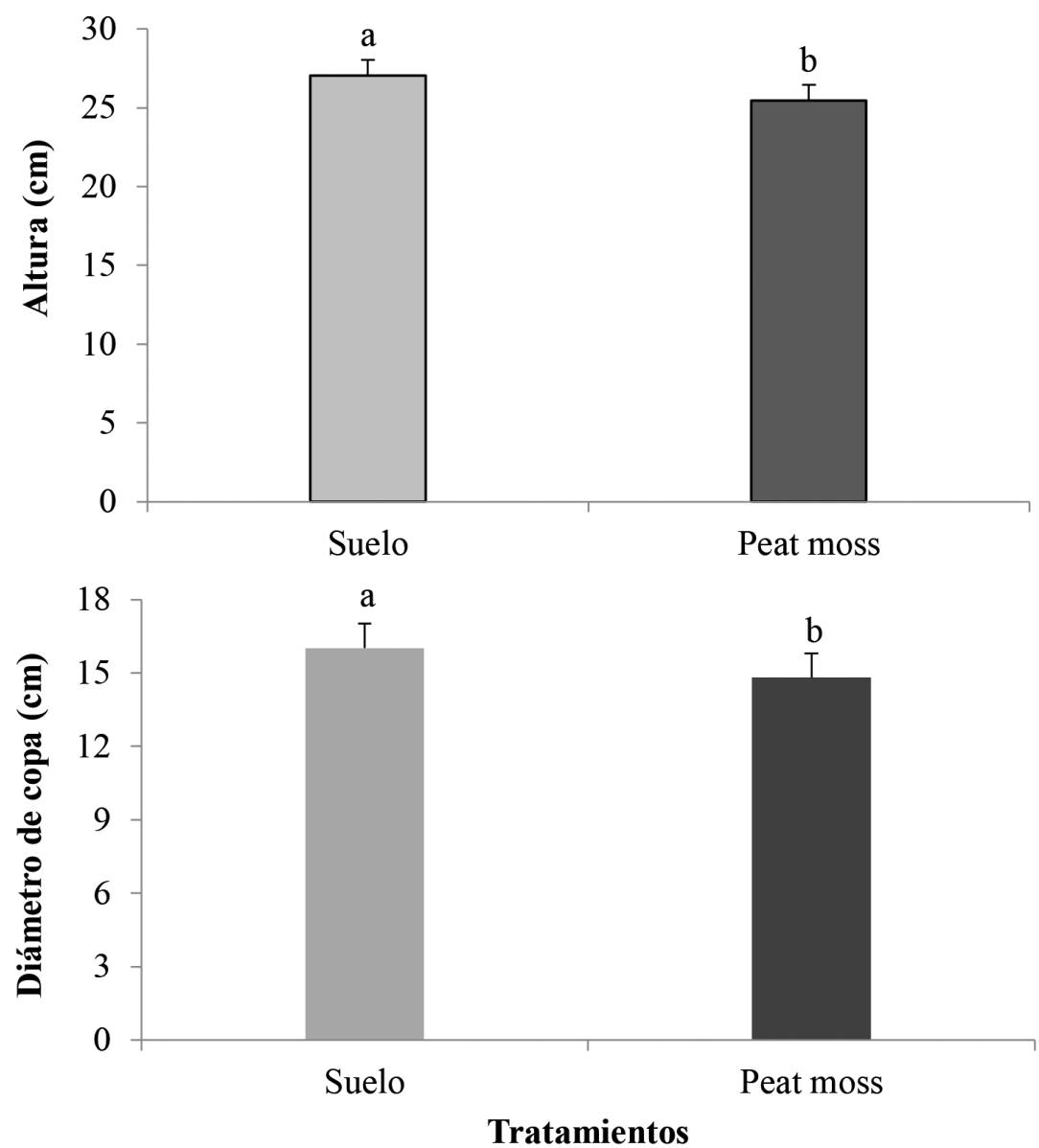

Figura 4. A) Medición de la altura de los esquejes de lavanda cultivada en el suelo. B) Medición del diámetro de plantas de lavanda cultivadas en el suelo.

Figura 5B). Las plantas de lavanda mostraron una adaptación importante a las condiciones climáticas. Además, las plantas producidas por esquejes no solo presentaron un crecimiento apical, sino que también tuvieron brotación de la parte basal generando nuevos brazos. Se evidenció que la planta de lavanda se adaptó fácilmente y empezó a desarrollarse satisfactoriamente mostrando crecimientos aceptables en la cobertura aérea (follaje), parte útil de la planta, debido a que las hojas de la planta de lavanda se utilizan para la extracción de aceite esencial (Hernández y Jibaja, 2017). Actualmente el aceite de lavanda va adquiriendo una gran importancia económica dentro de la industria cosmética y de aromaterapia a nivel mundial. Una ventaja de este tipo de cultivo sería que las plantas de lavanda producidas por esquejes fueron cultivadas sin fertilizantes químicos, y pueden catalogarse dentro de la modalidad de producción orgánica. De estas plantas de lavanda se podrá obtener aceite esencial libre de contaminantes químicos.

\section{Longitud de raíz principal y número de raíces secundarias}

No se presentaron diferencias significativas $(p>0,05)$ con respecto al sustrato utilizado (suelo y pet moss) en la longitud de las raíces y en el número de raíces secundarias. Sin embargo, se estableció una correlación positiva entre la longitud de las raíces y el tamaño del esqueje $(r=0,983, p<0,001)$, indicando que se produce una dependencia entre la longitud radicular y el tamaño del esqueje, con un crecimiento radicular del 96\%. Sin embargo, los resultados obtenidos en este estudio no coinciden 

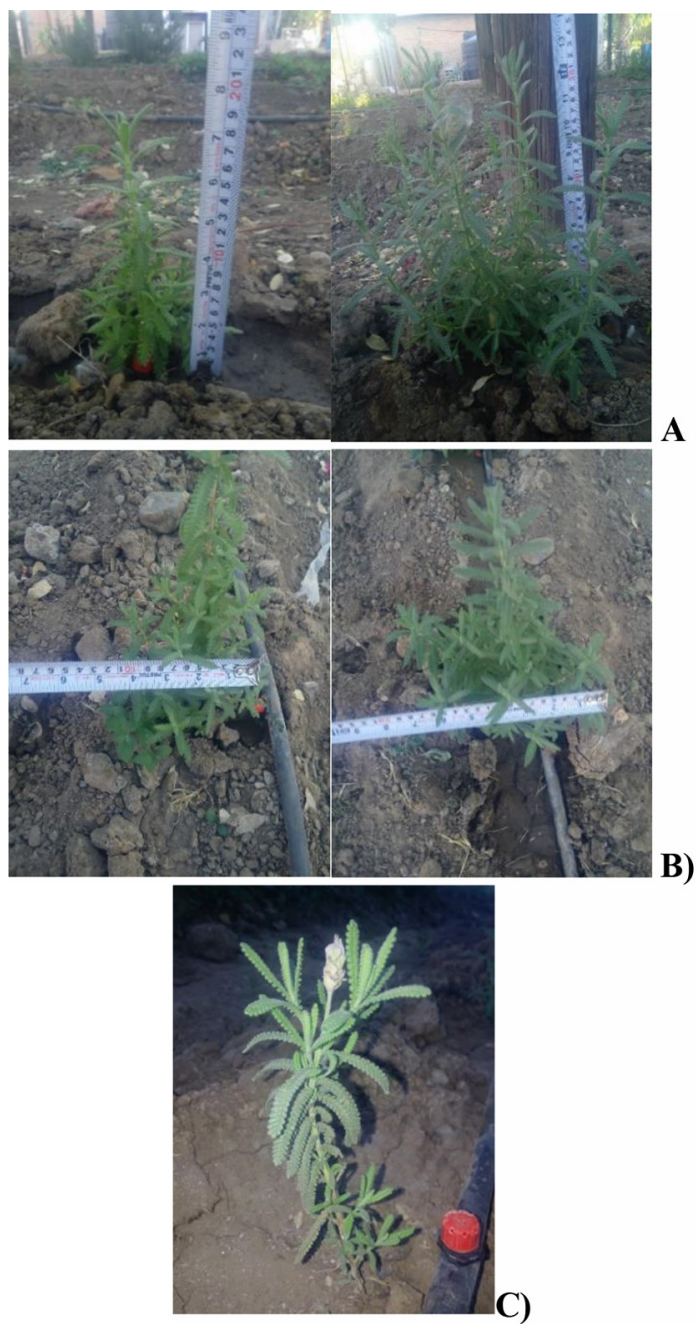

Figura 5. A) Medición de la altura de los esquejes de lavanda cultivada en el suelo. B). Medición del diámetro de plantas de lavanda cultivadas en el suelo. C) Floración de lavanda cultivada en el suelo.

con lo reportado por Bonfil-Sanders et al. (2007), quienes evaluaron esquejes de siete géneros de Bursera, y no establecieron correlación positiva entre cantidad de raíces y tamaño de esquejes. Esto puede deberse a que las burseras son árboles, mientras que la lavanda es un arbusto de talla baja. Además las condiciones de sustrato y temperatura utilizadas en cada estudio fueron diferentes. Se estableció una correlación positiva entre la longitud de las raíces secundarias y el tamaño de las raíces principales $(\mathrm{r}=0,88, p<0,001)$, lo cual indica que influye directamente la longitud radicular principal con el número de raíces secundarias presentando una producción de raíces adventicias del $88 \%$.
De acuerdo a Hernández et al. (2005), los esquejes de mayor tamaño en caña flecha (Gynerium sagittatumaub 1.) son los que presentan mayor longitud de raíces adventicias, por tener un alto contenido de materiales de reserva disponibles para el crecimiento de las raíces. Por lo tanto, el desarrollo del sistema radical de las plantas se afecta directamente por las condiciones de humedad, aireación, temperatura y resistencia mecánica en los suelos (Callejas et al., 2009). Así, los sistemas de riego pueden afectar la distribución de las raíces. El buen manejo de los factores productivos impactará directamente en la calidad del sistema radical y con ello el potencial productivo de las plantas (Davies y Zhang, 1991; Glenn, 2000; Ruiz, 2007). Es de gran importancia contar con plantas fuertes que presenten un buen sistema radicular, lo que contribuirá a tener plantas de buena talla, con un diámetro de copa apropiado y sobre todo un tallo firme (pie de la planta) y que deberá soportar su peso (Mc Caughey-Espinoza et al., 2019). Por lo anterior, es necesario señalar que la agricultura hoy en día requiere de otras alternativas de producción y dada la importancia del crecimiento paulatino de la producción orgánica y los beneficios que ofrecen las plantas aromáticas y medicinales por sus compuestos fitoquímicos, se recomienda estudiar a mayor profundidad el comportamiento de la lavanda (Lavandula angustifolia Mill) en condiciones semiáridas, debido a que existe escasa información al respecto. Este trabajo de investigación contribuye con información novedosa y relevante acerca del cultivo de lavanda (Lavandula angustifolia Mill), que puede dar una pauta importante para generar áreas de oportunidades en el plano agrícola e industrial al presentar un potencial productivo en el noroeste de México, en condiciones bajo sombra.

\section{Plagas y enfermedades}

En el periodo de enraizamiento de los esquejes, así como en su etapa de climatización y establecimiento de las plántulas en el área de estudio, no se presentaron plagas ni ningún tipo de enfermedad. Esto se puede atribuir a que dicha área no tiene otro tipo de plantaciones que pudieran atraer plagas. Además esta fue la primera plantación de lavanda (Lavandula angustifolia Mill) en esa zona de estudio. 


\section{Conclusiones y recomendaciones}

Sembrar los esquejes de lavanda (Lavandula angustifolia Mill) directamente en el suelo produjo un mayor enraizamiento comparado con los esquejes sembrados en pet moss. La longitud de los esquejes utilizada en este estudio permitió producir nuevas plántulas de lavanda, las cuales florecieron después de un mes de su trasplante en suelo y pet moss, asegurando la producción de semilla de lavanda. Se obtuvieron esquejes con un alto porcentaje de enraizamiento sin el uso de fitohormonas o sustrato especial (fertilizante químico), enraizando esquejes directamente en el suelo. La propagación de lavanda por esquejes fue exitosa a la intemperie y, por lo tanto, no se requiere de invernadero para obtener plantas con un alto porcentaje de enraizamiento. La capacidad de enraizamiento y la longitud de los esquejes afectaron la cantidad de raíces emitidas. Después de cuatro meses de trasplantar los esquejes de lavanda se logró producir plantas con un alto porcentaje de follaje, el cual se utiliza para la extracción de aceite de lavanda. Para futuras investigaciones se recomienda usar esquejes de lavanda con una longitud de $12 \mathrm{~cm}$ que presenten más cantidad de meristemos para la producción de raíces, lo que es ideal para asegurar el trasplante de las nuevas plantas de lavanda. Además, se sugiere realizar un análisis de porcentaje de obtención de aceite esencial de lavanda de las plantas. Por lo tanto, la lavanda crecida en suelo bajo malla sombra puede ser un cultivo con gran potencial productivo para el noroeste de México.

\section{Literatura Citada}

Azizi, M.; Bos, R.; Woerdenbag, H.J.; Kayser, O.

2006. A comparative study of four chamomile cultivars cultivated in Iran. In I International Symposium on Chamomile Research, Development and Production, 749: 93-96 p.

Bandurski, R.; Schulze, A.; Jensen, P.; Desrosiers, M.; Epel

B.; Kowalczyk, S.

1993. The mechanism by which an asymmetric distribution of plant growth hormone is attained. Adv. Space Res, 12 (1): 203-210.

Bonfil-Sanders, C.; Mendoza-Hernández, P.E.; Ulloa-Nieto, J.A. 2007. Enraizamiento y formación de callos en estacas de siete especies del género Bursera. Agrociencia, 41 (1): 103-109.

Celedón, N.A.; Lerdon, J.A.

2014. Economic evaluation of the establishment of a Lavandula angustifolia crop in Pucón, Región de la Araucanía, Chile. Rev. Agro Sur, 42 (1): 63-71.

Callejas, R.R.; Canales, P.P.; de Cortázar, V.G.G.D.

2009. Relationship between root growth of "Thompson Seedless" grapevines and soil temperature. Chilean J. Agric. Res, 69 (4): 496-502.

Davies, W.J.; Zhang, J.

1991. Root signals and the regulation of growth and development of plants in drying soil. Annual review of plant biology, 42 (1): 55-76.

Dole J.M.; Gibson J.L.

2006. Cutting propagation: A guide to propagation and producing floriculture crops. In: Dole; J.M.; Gibson J.L. (eds). Propagation Basics. Ball Publishing. Batavia, Illinois, USA. pp. 3-16.

Garay, C.R.E.; Echeverría, F.M.C.

2013. Propagación vegetativa de Jatropha curcas L. por estacas. Investigación Agraria, 12 (2): 69-73.

Glenn, D.M.

2000. Physiological effects of incomplete root-zone wetting on plant growth and their implications for irrigation management. HortScience, 35 (6): 1041-1043.
Hartmann, H.T.; Kester, D.E.; Davies, F.T.; Geneve, R.L. 2011. Hartmann \& Kester's Plant propagation: principles and practices. 8 eds. Prentice Hall. 915 p.

Hernández, J.R.; Tatis, H.A.; Ayala, C.E.C.

2005. Influencia del ácido indolbutírico y ácido naftalenoacético sobre el enraizamiento de esquejes de caña flecha (Gynerium sagittatumaubl.). Temas agrarios, 10 (1): 5-13.

Hernández, H.I.C.; Jibaja C.M.

2017. Producción y exportación de aceites esenciales de plantas aromáticas de ciclo corto. Revista Observatorio de la Economía Latinoamericana, Ecuador, ISSN: 1696-8352. Disponible en: http://www.eumed.net/cursecon/ecolat/ aceites-esenciales-ecuador.htmlConsultado: 11/oct/2019.

Herrera, J.G.Á.; Rodríguez, S.L.; Chacón, E.

2007. Efecto de diferentes tamaños de esqueje y sustratos en la propagación del romero (Rosmarinus officinalis L.). Agronomía Colombiana, 25 (2): 224-230.

Juárez-Rosete, C.R.; Aguilar-Castillo, J.A.; Juárez-Rosete, M.E.; Bugarín-Montoya, R.; Juárez-López, P.; Cruz-Crespo E. 2013. Hierbas aromáticas y medicinales en México. Revista Bio Ciencias, 2(3): 119-129.

Kochhar, S.; Singh, S.P.; Kochhar, V.K.

2008. Effect of auxins and associated biochemical changes during clonal propagation of the biofuel plant-Jatropha curcas. Biomass and Bioenergy, 32 (12): 1136-1143.

Lemes-Hernández, C.M.; Rodríguez-Ferradá, C.A.; Acosta de la Luz, L.

2001. Multiplicación vegetativa de Rosmarinus officinalis L. (romero). Revista Cubana de Plantas Medicinales, 6 (3): 79-82.

Marschner, $\mathrm{H}$.

2002. Mineral nutrition of higher plants. 2nd. eds. Editorial Academic Press. London, UK. 889 p.

Meza-Sánchez, R.; Osuna-Leal, E.

2003. Estudio dasométrico del mezquite en la zona de Las Pocitas BCS INIFAP. Todos Santos, BCS México. Folleto científico, 3: 1-7. 
Mc Caughey-Espinoza, D.M.; Ayala-Astorga, G.I.; VelázquezCaudillo, J.; Anaya-Islas, J.; Canseco-Vilchis, E.

2017. Creación de un jardín botánico y de árbol madre de arbustivas forrajeras nativas del estado de Sonora. Idesia (Arica), 35 (4): 35-45.

Mc Caughey-Espinoza, D.M.; Ayala-Astorga, G.I.; BurboaZazueta, M.G.; Retes-López, R.; Ochoa-Meza, A.

2019. Uso de plantas nativas para la rehabilitación de canteras en Sonora. Idesia (Arica), 36 (4): 17-24.

Ocampo, R.A.; Valverde, R.

2000. Manual de cultivo y conservación de plantas medicinales. TRAMIL. San José, Costa Rica. 148 p.

Ordóñez, C.; Gómez, H.; Jurado, H.R.O.; Burbano, T.C.L.

2012. Evaluación de un sistema de propagación vegetativa mediante esquejes en lulo silvestre Solanum hirtum Vahl, S. marginatum Lf, S. sessiliflorum Dun, S. mammosum L. y S. umbellatum Mill. Revista de Ciencias Agrícolas, 29 (1): 29-41.
Ruiz, R.; Sellés, G.; Ahumada, R.

2007. Aspectos físicos del suelo y calidad de fruta en parronales de uva de mesa. Manejo de suelo y riego en vides para vino y mesa. Serie Actas, 39: 101-114.

Ruiz-Solsol, H.; Mesén, F.

2010. Efecto del ácido indolbutírico y tipo de estaquilla en el enraizamiento de sacha inchi (Plukenetia volubilis L.). Agronomía Costarricense, 34 (2): 269-285.

Salisbury, F.; Ross, C.

1994. Fisiología vegetal. Grupo Editorial Iberoamérica, México D.F. 759 p.

Solís, C.A.; Jiménez, V.; Arias, J.

2015. Propagación asexual de azul de mata (Justicia tinctoria (Oerst.) DN Gibson, Fam. Acanthaceae) por medio de estacas. Agronomía Costarricense, 39 (2): 91-103.

Pérez, C.R.

2009. Frutas y hortalizas orgánicas de la red de mercados y tianguis orgánicos de México. Estudio del Sial. Claridades Agropecuarias, 194: 25-45. 
